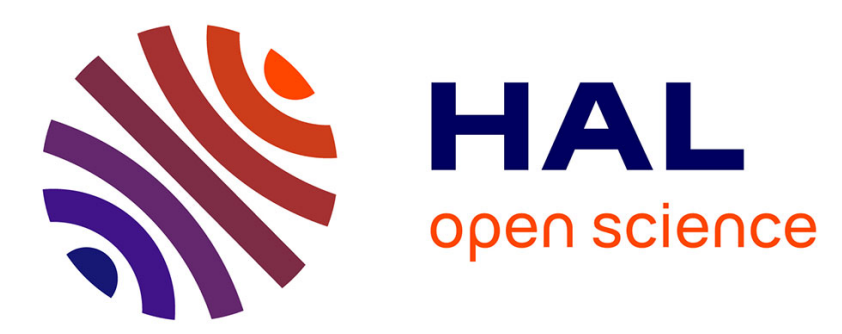

\title{
A New Differential Space-Time Modulation Scheme based on Weyl Group
}

Hui Ji, Gheorghe Zaharia, Jean-François Hélard

\section{To cite this version:}

Hui Ji, Gheorghe Zaharia, Jean-François Hélard. A New Differential Space-Time Modulation Scheme based on Weyl Group. International Symposium on Signals, Circuits and Systems, Jul 2013, Iasi, Romania. pp.1-4. hal-00873872

\section{HAL Id: hal-00873872 https://hal.science/hal-00873872}

Submitted on 16 Oct 2013

HAL is a multi-disciplinary open access archive for the deposit and dissemination of scientific research documents, whether they are published or not. The documents may come from teaching and research institutions in France or abroad, or from public or private research centers.
L'archive ouverte pluridisciplinaire HAL, est destinée au dépôt et à la diffusion de documents scientifiques de niveau recherche, publiés ou non, émanant des établissements d'enseignement et de recherche français ou étrangers, des laboratoires publics ou privés. 


\title{
A New Differential Space-Time Modulation Scheme based on Weyl Group
}

\author{
Hui Ji, Gheorghe Zaharia, Jean-François Hélard \\ Université Européenne de Bretagne (UEB) \\ INSA, IETR, UMR 6164, F-35708, Rennes, France \\ Email:\{hui.ji; gheorghe.zaharia; jean-francois.helard\}@insa-rennes.fr
}

\begin{abstract}
In this paper, a new differential space-time modulation (DSTM) scheme for $4 \times 4$ multiple input multiple output (MIMO) systems is proposed. This scheme is used for MIMO systems where the channel coefficients are not available at both the transmitters and the receivers. The transmission matrix used in this scheme is based on the Weyl group. Simulation results show that this new scheme with four transmit antennas outperforms the well-known Tarokh's differential space-time block coding (DSTBC) scheme. The spectral efficiency of this scheme can be up to $3 \mathrm{bps} / \mathrm{Hz}$.
\end{abstract}

Keywords-MIMO, DSTM, DSTBC, non-coherent.

\section{INTRODUCTION}

In the late 1990s, due to the large demands of wireless spectral resource, researchers resort to multiple antennas to enlarge the capacity and robustness of wireless communication systems. Huge amounts of studies have been carried out on this domain. Reference [1], [2] analyzed the capacity and the error exponents of such systems in the presence of Gaussian noise and got some fundamental theoretical results. Generally, people design space-time coding/modulation schemes to get the requirements. At the beginning, when the throughput was not so high, people could estimate the channel coefficients easily. Several schemes with known channel state information (CSI) have been widely analyzed.

However, the estimation of CSI consumes much time or frequency resource, especially when the number of antennas is large or the propagation channel changes rapidly. As the number of transmit antennas grows, the training time to probe the channel grows proportionately [3], which in turn reduces the overall system throughput. Therefore, solutions that do not require channel information are very interesting. Marzetta and Hochwald [4] analyzed the capacity of the MIMO systems in such cases. Based on the results, they proposed the unitary space-time modulation scheme (USTM) [5]. Analogous to single antenna DPSK (differential phase-shift keying) systems, Hochwald and Sweldens proposed the differential space-time modulation scheme (DUSTM) [6]. These two schemes are difficult to design, and furthermore there are not general design criteria for these two schemes. At the same time, Tarokh and Jafarkhani proposed a differential space-time block coding scheme [7] which is based on Alamouti's diversity scheme [8], and soon they expanded this scheme to systems with 4 transmit antennas [9]. This scheme is just suitable for MIMO systems with less than 4 transmit antennas and the spectral efficiency is limited. In [10], [11], the authors proposed a new kind of non-coherent space-time modulation scheme-matrix coded modulation (MCM) which is suitable for $2 \times 2$ MIMO systems. In the study of the MCM scheme, we found that the group used in this scheme can be applied in differential MIMO schemes.

In this paper, we propose a new differential space-time modulation scheme which is suitable for MIMO systems with 4 transmit antennas. The simulation results demonstrate the advantages of this new scheme over Tarokh's DSTBC scheme. Furthermore, the modulation of Tarokh's scheme with 4 transmit antennas can only be BPSK [9], which prevents the scheme to expand to higher spectral efficiency. Our scheme with four transmit antennas can be extended to $3 \mathrm{bps} / \mathrm{Hz}$. And the bit error rate performance can be improved with the method of searching the best distance spectrum.

The following notations are used through the paper: $\operatorname{Tr}\{A\}$ denotes the trace of the matrix $A$ and $A^{H}$ denotes the conjugate transpose of $A$. $\|A\|$ means the Frobenius norm of $A$, i.e., $\|A\|=\sqrt{\sum_{i, j}\left|a_{i j}\right|^{2}}=\sqrt{\operatorname{Tr}\left\{A^{H} A\right\}} \cdot \operatorname{Re}\{z\}$ is the real part of the complex number $z$. The sign $\lfloor K\rfloor$ denotes the nearest integer number less than $\mathrm{K}$, and $\otimes$ denotes the Kronecker product. The zero-mean, unit-variance, circularly symmetric, complex Gaussian distribution is denoted as $C N(0,1)$.

\section{Differential MiMO System Model}

The MIMO system model in the matrix form is:

$$
Y_{\tau}=H_{\tau} X_{\tau}+W_{\tau}
$$

where $\tau$ is the time index. $H_{\tau}$ is the channel coefficient matrix at time $\tau$ and its size is $M \times N$, where $M$ is the number of transmit antennas and $N$ is the number of receive antennas. The element $h_{n m}$ is the path gain of the quasi-static channel from the transmit antenna $m$ to the receive antenna $n$, and follows complex Gaussian distribution with zero-mean and unit-variance, i.e., $h_{n m} \sim C N(0,1) . X_{\tau}$ is the $M \times T$ transmission matrix, where $T$ denotes the normalized symbol durations of each matrix, i.e., on each transmit antenna, $T$ symbols are transmitted. $Y_{\tau}$ is the received matrix. $W_{\tau}$ is the additive white Gaussian noise matrix, $w_{n t} \sim C N\left(0, \sigma^{2}\right)$ and $\sigma^{2}$ is the power of the noise. We denote the spectral efficiency of the system as $R$ expressed in $\mathrm{bps} / \mathrm{Hz}$.

In this paper, we assume that the channel coefficients are constant during $L$ symbols. Therefore, we ignore the index $\tau$ 
of the matrix $H_{\tau}$ and the channel matrix is written to be $H$.

Furthermore, we assume a normalized power over $M$ transmit antennas:

$$
\sum_{m=1}^{M}\left|x_{m t}\right|^{2}=1, t=1, \ldots, T
$$

As it is proved in [4], for non-coherent MIMO systems, for any block length $T$, any number of receive antennas $N$ and any SNR (signal to noise ratio), the capacities obtained with $M>T$ and $M=T$ are equal. Therefore, we choose $M=T$ in our study.

\section{A. Differential space-time modulation}

In the differential space-time modulation systems, one block of information bits is mapped onto a matrix in the candidate set $P$. For example, the transmitter send a reference matrix $X_{0}=V_{0}$ at time $\tau_{0}$. The first block of the information bits is mapped to the matrix $V_{\tau_{1}}$ and the second block is mapped to $V_{\tau_{2}}$ etc. The fundamental differential transmission relation is:

$$
X_{\tau+1}=X_{\tau} V_{i_{\tau+1}}, \tau=0,1, \ldots
$$

Therefore, the sequence of transmitted matrices is:

$$
\begin{aligned}
& X_{0}=V_{0} \\
& X_{1}=X_{0} V_{i_{1}}=V_{0} V_{i_{1}} \\
& \ldots \\
& X_{\tau}=X_{\tau-1} V_{i_{\tau}}=V_{0} V_{i_{1}} \ldots V_{i_{\tau}}
\end{aligned}
$$

At the receiver side, the antennas received a matrix stream $Y_{0}, \ldots, Y_{\tau}, Y_{\tau+1}, \ldots$ We know that

$$
\begin{aligned}
Y_{\tau} & =H X_{\tau}+W_{\tau} \\
Y_{\tau+1} & =H X_{\tau+1}+W_{\tau+1}
\end{aligned}
$$

Based on the differential transmission equation (3), we get

$$
\begin{aligned}
Y_{\tau+1} & =H X_{\tau+1}+W_{\tau+1} \\
& =Y_{\tau} V_{i_{\tau+1}}+W_{\tau+1}-W_{\tau} V_{i_{\tau+1}} \\
& =Y_{\tau} V_{i_{\tau+1}}+W_{\tau+1}^{\prime}
\end{aligned}
$$

where $W_{\tau+1}^{\prime}=W_{\tau+1}-W_{\tau} V_{i_{\tau+1}}$.

Therefore, to estimate the information matrix, the maximum likelihood demodulator is

$$
\begin{aligned}
\hat{V}_{i_{\tau+1}} & =\arg \min _{V \in P}\left\|Y_{\tau+1}-Y_{\tau} V\right\| \\
& =\arg \min _{V \in P} \operatorname{Tr}\left\{\left(Y_{\tau+1}-Y_{\tau} V\right)^{H}\left(Y_{\tau+1}-Y_{\tau} V\right)\right\} \\
& =\arg \min _{V \in P} \operatorname{Tr}\left\{\operatorname{Re}\left(Y_{\tau+1}^{H} Y_{\tau} V\right)\right\}
\end{aligned}
$$

\section{New Differential Space-Time Modulation SCHEME}

\section{A. The constellation of the new scheme}

In our scheme, the transmitted matrices are based on the Weyl group used in [10], [11]. The Weyl group $G_{w}$ is a set that contains 12 cosets $\left(C_{0}, C_{1}, \ldots, C_{11}\right)$. Each coset contains 16 invertible matrices. The first coset is defined as:

$$
C_{0}=\left\{\alpha\left[\begin{array}{ll}
1 & 0 \\
0 & 1
\end{array}\right], \alpha\left[\begin{array}{cc}
1 & 0 \\
0 & -1
\end{array}\right], \alpha\left[\begin{array}{ll}
0 & 1 \\
1 & 0
\end{array}\right], \alpha\left[\begin{array}{cc}
0 & 1 \\
-1 & 0
\end{array}\right]\right\}
$$

with $\alpha \in\{1,-1, i,-i\}$. The 12 cosets of $G_{w}$ are derived from $C_{0}$ as follows:

$$
C_{k}=A_{k} C_{0}, \forall k=0,1, \ldots, 11
$$

where the matrices $A_{k}, k=0,1, \ldots, 5$ are respectively:

$$
\begin{array}{r}
A_{0}=\left[\begin{array}{ll}
1 & 0 \\
0 & 1
\end{array}\right], A_{1}=\left[\begin{array}{ll}
1 & 0 \\
0 & i
\end{array}\right], A_{2}=\frac{1}{\sqrt{2}}\left[\begin{array}{cc}
1 & 1 \\
1 & -1
\end{array}\right], \\
A_{3}=\frac{1}{\sqrt{2}}\left[\begin{array}{cc}
1 & 1 \\
i & -i
\end{array}\right], A_{4}=\frac{1}{\sqrt{2}}\left[\begin{array}{cc}
1 & i \\
1 & -i
\end{array}\right], A_{5}=\frac{1}{\sqrt{2}}\left[\begin{array}{ll}
1 & i \\
i & 1
\end{array}\right],
\end{array}
$$

and the matrices $A_{k}, k=6,7, \ldots, 11$ are given by:

$$
A_{k+6}=\eta A_{k}, \text { with } \quad \eta=(1+i) / \sqrt{2}, \forall k=0,1, \ldots, 5
$$

Remind that, there are 192 matrices in this group, and we number the matrices as $M_{0}, M_{1}, \ldots, M_{191}$. Furthermore, they are all unitary matrices, i.e., the inverse of the matrix is equal to the conjugate transpose of the matrix.

We define the distance between two matrices $M_{a}$ and $M_{b}$ as:

$$
D_{a, b}=\left\|M_{a}-M_{b}\right\|
$$

We can see that $D_{a, b}=D_{b, a}$. Therefore, there are $191 \times$ $192 / 2=18336$ values $D_{a, b}$ with $0 \leq a<b \leq 191$. For any value $a$, the distribution of the 191 values $D_{a, b}$ with $b \neq a$ is the same, as shown in Fig. 1(a). For $C_{0}$, this distribution is given in Fig. 1(b). As in [12]-[14], these results are used to optimize the BER performance.

\section{B. Differential transmission and reception}

Consider a MIMO system with $M=2$ transmit antennas and $N=2$ receive antennas. Each transmit matrix is sent during $T=2$ symbol durations. The number of receive antennas is arbitrary, i.e., we can set $N=1,2,3, \ldots$ We use the coset $C_{0}$ as the candidate mapping matrices set. There are 16 matrices in the coset $C_{0}$, say $M_{0}, M_{1}, \ldots, M_{15}$, so it is possible to map 4 bits to a given matrix of the coset $C_{0}$. Thus, 4 bits are sent during 2 symbol durations. Hence, the spectral efficiency $R$ is $2 \mathrm{bps} / \mathrm{Hz}$.

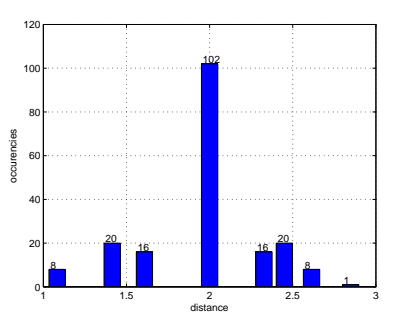

(a) Weyl group

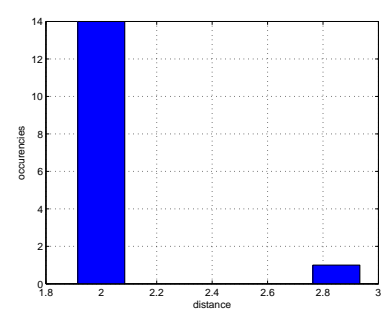

(b) Coset $C_{0}$
Fig. 1. Distance spectrum 
At time $\tau=0$, we transmit a reference matrix $X_{0}=M_{0}=$ $\left[\begin{array}{ll}1 & 0 \\ 0 & 1\end{array}\right]$.

Suppose that at time $\tau, X_{\tau}$ is transmitted. At time $\tau+1$, a block of 4 bits arrives. These bits are mapped onto one of the matrices $M_{i_{\tau+1}}=M_{a}$ of the coset $C_{0}$, and then

$$
X_{\tau+1}=X_{\tau} M_{i_{\tau+1}}
$$

is transmitted.

The demodulation procedure is shown in the Section II.

As there are $K=192$ matrices in the Weyl group $G_{w}$, for MIMO systems with 2 transmit antennas, the maximum spectral efficiency we can get is $R=\frac{1}{M}\left\lfloor\log _{2} K\right\rfloor=3.5$ bps/Hz.

\section{MIMO systems with 4 transmit antennas}

To design a MIMO system with 4 transmit antennas, the Kronecker product is used to expand the Weyl group.

With the assumption $M=T$ defined in section II, for MIMO systems with 4 transmit antennas, $4 \times 4$ transmit matrices are used.

Using the Kronecker product between each couple of $2 \times 2$ matrices of the Weyl group, $4 \times 4$ matrices are obtained. Only 4608 matrices are distinct. They are denoted $N_{0}, N_{1}, \ldots, N_{4607}$. The set of these matrices is also a group denoted $G_{w 4}$.

In our first proposal which is used for $R=1 \mathrm{bps} / \mathrm{Hz}$, we make the Kronecker products between the first matrix $M_{0}=$ $\left[\begin{array}{ll}1 & 0 \\ 0 & 1\end{array}\right]$ of $C_{0}$ and all the matrices in $C_{0}$ to get a set $C_{00}$.

$$
\begin{gathered}
C_{00}=M_{0} \otimes C_{0} \\
C_{00}=\left\{\alpha\left[\begin{array}{llll}
1 & 0 & 0 & 0 \\
0 & 1 & 0 & 0 \\
0 & 0 & 1 & 0 \\
0 & 0 & 0 & 1
\end{array}\right], \alpha\left[\begin{array}{cccc}
1 & 0 & 0 & 0 \\
0 & -1 & 0 & 0 \\
0 & 0 & 1 & 0 \\
0 & 0 & 0 & -1
\end{array}\right],\right. \\
\left.\alpha\left[\begin{array}{llll}
0 & 1 & 0 & 0 \\
1 & 0 & 0 & 0 \\
0 & 0 & 0 & 1 \\
0 & 0 & 1 & 0
\end{array}\right], \alpha\left[\begin{array}{cccc}
0 & 1 & 0 & 0 \\
-1 & 0 & 0 & 0 \\
0 & 0 & 0 & 1 \\
0 & 0 & -1 & 0
\end{array}\right]\right\}
\end{gathered}
$$

where $\alpha \in\{1,-1, i,-i\}$.

Four information bits are viewed as a block. The block is mapped onto one of the 16 matrices of $C_{00}$ as an information matrix. Once the matrix $V_{i_{\tau}}$ is obtained, it is used to compute the current transmit matrix $X_{\tau}=X_{\tau-1} V_{i_{\tau}}$. There are 4 information bits transmitted during 4 symbol-durations, thus the spectral efficiency is $R=1 \mathrm{bps} / \mathrm{Hz}$.

The constellation of the modulation of this scheme (i.e., the possible value of the matrices' elements) is $\{ \pm 1, \pm i, 0\}$ which corresponds to $Q P S K \cup 0$, and the spectral efficiency is $1 \mathrm{bps} / \mathrm{Hz}$. For comparison, the $4 \times 4$ DSTBC scheme [12] with modulation BPSK has the same spectral efficiency. However, this new scheme has better BER performance. Furthermore, there are $K=4608$ distinct matrices in the group $G_{w 4}$. The maximum spectral efficiency we can get is
$R=\frac{1}{M}\left\lfloor\log _{2} K\right\rfloor=\frac{1}{4}\left\lfloor\log _{2} 4608\right\rfloor=3 \mathrm{bps} / \mathrm{Hz}$. The simulation results with different spectral efficiencies are shown in Fig.4.

For the spectral efficiency $R=2,8$ bits should be transmitted in 4 symbol-durations. The information bits are mapped into one of the $2^{8}=256$ matrices. We select the first 256 matrices from $G_{w 4}$ as the candidate transmission set. For $R=3$, we should transmit 12 bits in 4 symbol-durations. Similarly, we select the first 4096 matrices from $G_{w 4}$ as the candidate transmission set. The selection of the matrices is arbitrary.

As the method used for 2 transmit antennas, we can also resort to the distance spectrum to improve the BER performance.

\section{Simulation Results}

In this section, we present several simulation results of the new differential space-time modulation scheme compared to some existing differential space-time modulation schemes. We assume that the channel is quasi-static, i.e., the channel coefficients do not change during one frame of transmission matrices. In our simulation, the length of the frame is $L=100$ (i.e., the channel coefficients keep constant during 100 symbol durations and change to new independent realizations for the next frame).

We find that for MIMO systems with 2 transmit antennas, our new scheme performs worse than the Tarokh's DSTBC scheme [7], as shown in Fig. 2. This is because the decoding method of our scheme is a general maximum likelihood decoding without any pre-process, while the variable used to decode in [7] is linearly scaled by the channel coefficients due to some pre-process. However, this new scheme performs better than the corresponding DUSTM scheme [6] when SNR is less than $14 \mathrm{~dB}$. This is because the DUSTM is designed for large SNR environments.

Using the distance spectrum of the Weyl group, a new set $S=C_{0}^{r} \cup A_{3} C_{0}^{r}$ is considered as an alternative to $C_{0}$. The set $C_{0}^{r}$ contains the 8 real matrices of $C_{0}$ and $A_{3} C_{0}^{r}$ is the set obtained by multiplying $A_{3}$ with the matrices of $C_{0}^{r}$. As the set $C_{0}$, the set $S$ contains 8 couples $\left(M_{a}, M_{b}\right)$ with $D_{a, b}=\left\|M_{a}-M_{b}\right\|=2.8284$, the greatest distance between 2 matrices of $G_{W}$. If we consider 2 couples $\left(M_{a}, M_{b}\right)$ and $\left(M_{c}, M_{d}\right)$ of $C_{0}$, with $D_{a, b}=D_{c, d}=2.8284$, we have $D_{a, c}=D_{a, d}=D_{b, c}=D_{b, d}=2$, while for the set $S$, if $D_{a, b}=D_{c, d}=2.8284$, then $\left[\begin{array}{llll}D_{a, c} & D_{a, d} & D_{b, c} & D_{a, d}\end{array}\right]=$ [1.4142 1.4142 2.4495 2.4495]. As shown in Fig.2, the results obtained for $C_{0}$ and $S$ are similar.

Fig. 3 shows that, for 4-transmit-antenna systems, this new system performs better than the original Tarokh's differential STBC [9]. On the bit error rate level of $10^{-4}$, it's about 0.57 $\mathrm{dB}$ better than [9]. And this new scheme performs a little worse than the DUSTM scheme for large SNR.

Fig. 4 shows the BER performance of the new scheme with different spectral efficiency. It shows that with the spectral efficiency up to $\mathrm{R}=3 \mathrm{bps} / \mathrm{Hz}$, the new scheme performs well enough. However, Tarokh's DSTBC scheme can not be expanded to $2^{b}$-PSK $(M \geq 4)$ modulations, which prevent it 


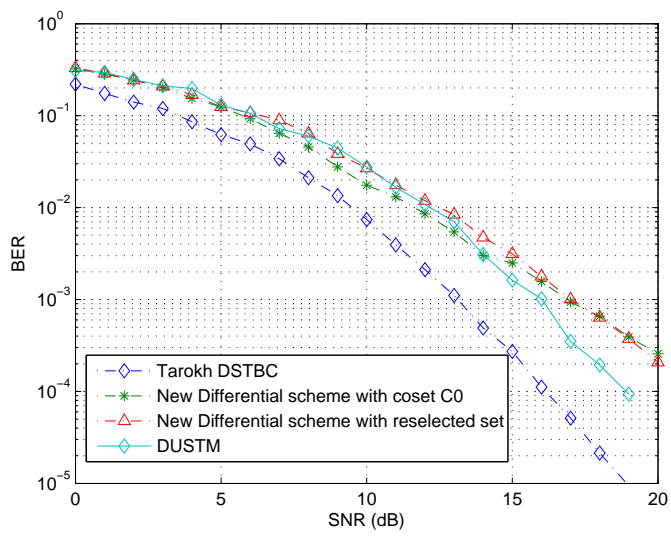

Fig. 2. Simulation results of DSTBC [7], [9], DUSTM [6] and our new differential space-time modulation scheme $(\mathrm{M}=2, \mathrm{~N}=2, \mathrm{R}=2)$.

to achieve higher spectral efficiency. Furthermore, considering the distance spectrum, we can get a better set for this scheme. As shown in Fig. 4, the new set selected for $\mathrm{R}=2$ scheme has a better distance spectrum. In fact, we select the first 16 matrices from every successive 192 matrices of the group $G_{w 4}$, i.e., the set is

$$
\begin{aligned}
& \left\{N_{0}, N_{1}, \ldots, N_{15},\right. \\
& N_{192}, N_{193}, \ldots, N_{207}, \\
& N_{384}, N_{385}, \ldots, N_{399}, \\
& \left.\ldots, N_{2880}, \ldots, N_{2895}\right\} . \\
& \text { V. CONCLUSION }
\end{aligned}
$$

\section{CONCLUSion}

In this paper, we proposed a new differential space time modulation scheme based on the Weyl group for $4 \times 4 \mathrm{MIMO}$ systems. Simulation results show that this scheme outperforms the well-known Tarokh's differential STBC scheme. The spectral efficiency of this scheme can be up to $3 \mathrm{bps} / \mathrm{Hz}$, so higher than $1 \mathrm{bps} / \mathrm{Hz}$ of Tarokh's DSTBC scheme with four transmit antennas. Furthermore, considering the distance spectrum, the BER performance of our scheme can be improved. The new scheme can be extended to MIMO systems with more transmit antennas, say 8 or 16 .

\section{REFERENCES}

[1] E. Telatar, "Capacity of multi-antenna gaussian channels," European Transactions on Telecommunications, vol. 10, no. 6, pp. 585-596, 1995.

[2] G. J. Foschini and M. J. Gans, "On limits of wireless communications in a fading environment when using multiple antennas," Wireless Pers. Commun., vol. 6, pp. 311-335, 1998.

[3] B. Hassibi and B. M. Hochwald, "How much training is needed in multiple-antenna wireless links," IEEE Trans. Inform. Theory, vol. 49, pp. 951-963, 2003.

[4] T. L. Marzetta and B. M. Hochwald, "Capacity of a mobile multipleantenna communication link in rayleigh flat fading," IEEE Trans. Inform. Theory, vol. 45, no. 1, pp. 139-157, 1999.

[5] B. M. Hochwald and T. L. Marzetta, "Unitary space-time modulation for multiple-antenna communications in rayleigh flat fading," IEEE Trans. Inform. Theory, vol. 46, no. 2, pp. 543-564, 2000.

[6] B. M. Hochwald and W. Sweldens, "Differential unitary space time modulation," IEEE Trans. Commun., vol. 48, no. 12, pp. 2041-2052, 2000 .

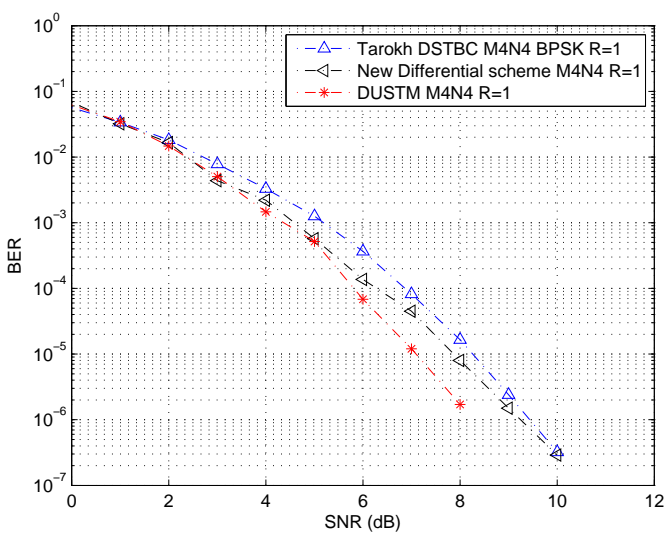

Fig. 3. Simulation results of DSTBC [7], [9], DUSTM [6] and our new differential space-time modulation scheme $(M=4, N=4, R=1)$.

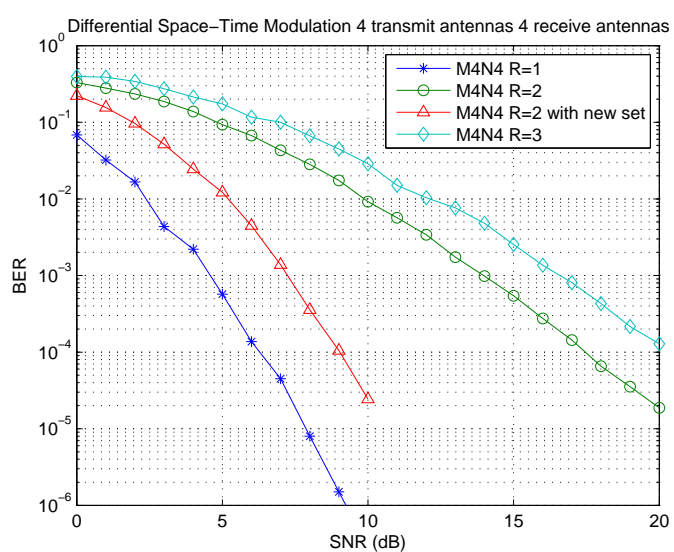

Fig. 4. Simulation results of the new differential space-time scheme for 4 transmi antennas and 4 receive antennas with spectral efficiency 1,2 and 3 $\mathrm{bps} / \mathrm{Hz}$ respectively.

[7] V. Tarokh and H. Jafarkhani, "A differential detection scheme for transmit diversity," IEEE J. Select. Areas Commun., vol. 18, no. 7, pp. 1169-1174, 2000.

[8] S. M. Alamouti, "A simple transmitter diversity scheme for wireless communications," IEEE J. Select. Areas Commun., vol. 16, no. 8, pp. 1451-1458, 1998.

[9] H. Jafarkhani and V. Tarokh, "Multiple transmit antenna differential detection from generalized orthogonal designs," IEEE Trans. Inform. Theory, vol. 47, no. 6, pp. 2626-2631, 2001.

[10] A. E. Arab, J.-C. Carlach, and M. Hélard, "A new non-coherent MIMO scheme: Matrix coded modulation 'MCM',' in International Symposium on Communications and Information Technologies(ISCIT), pp. 120-125, 2011.

[11] A. E. Arab, J.-C. Carlach, and M. Hélard, "Joint space-time coded modulation and channel coding for iterative non-coherent MIMO schemes," in Wireless Personal Multimedia Communications (WPMC), pp. 1-5, 2011.

[12] T. M. H. Ngo, G. Zaharia, S. Bougeard, and J.-F. Hélard, "4-PSK balanced STTC with two transmit antennas," in Proc. of VTC-Spring, pp. 1693-1697, 2007.

[13] T. M. H. Ngo, G. Zaharia, and J.-F. Hélard, "Balanced QPSK space-time trellis codes," Elect. Lett, vol. 44, no. 16, pp. 983-985, 2008.

[14] T. M. H. Ngo, G. Zaharia, S. Bougeard, and J.-F. Hélard, "A new class of balanced 4-PSK STTC for two and three transmit antennas," in Proc. of SPAWC, pp. 1-5, 2007. 